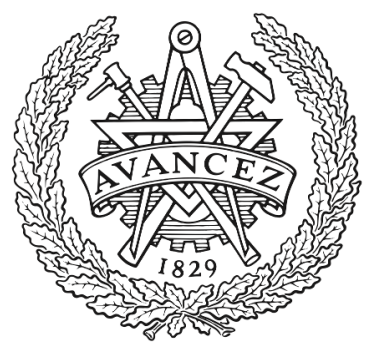

CHALMERS

UNIVERSITY OF TECHNOLOGY

\title{
Simulation of methane oxidation on Pt
}

Downloaded from: https://research.chalmers.se, 2023-04-26 12:55 UTC

Citation for the original published paper (version of record):

Zhdanov, V., Carlsson, P., Kasemo, B. (2007). Simulation of methane oxidation on Pt. Journal of Chemical Physics, 126: 234705-

N.B. When citing this work, cite the original published paper. 


\title{
Simulation of methane oxidation on $\mathrm{Pt}$
}

\author{
V. P. Zhdanov \\ Competence Centre for Catalysis, Chalmers University of Technology, S-412 96 Göteborg, Sweden \\ and Boreskov Institute of Catalysis, Russian Academy of Sciences, Novosibirsk 630090, Russia
}

\author{
P.-A. Carlsson and B. Kasemo \\ Competence Centre for Catalysis, Chalmers University of Technology, S-412 96 Göteborg, Sweden
}

(Received 12 March 2007; accepted 25 April 2007; published online 18 June 2007)

\begin{abstract}
The authors present a generic model of $\mathrm{CH}_{4}$ oxidation on $\mathrm{Pt}$ with the emphasis on the role of surface-oxide formation. The latter process is treated in terms of the theory of first-order phase transitions. The corresponding Monte Carlo simulations indicate that the surface-oxide formation may result in stepwise features in the reaction kinetics. Specifically, with increasing $\mathrm{CH}_{4}$ pressure and/or decreasing $\mathrm{O}_{2}$ pressure, the model predicts a sharp transition from a low-reactive state with the surface completely covered by oxide to a high-reactive state with the surface covered by chemisorbed oxygen. In the former case, the reaction is first order in $\mathrm{CH}_{4}$ and zero order in $\mathrm{O}_{2}$. In the latter case, both reaction orders are positive. All these findings help in interpreting available experiments. (C) 2007 American Institute of Physics. [DOI: 10.1063/1.2743403]
\end{abstract}

\section{INTRODUCTION}

The complete oxidation of methane on $\mathrm{Pt}$,

$$
\mathrm{CH}_{4}+2 \mathrm{O}_{2} \rightarrow \mathrm{CO}_{2}+2 \mathrm{H}_{2} \mathrm{O}
$$

is of considerable intrinsic interest because it is one of the generic catalytic reactions. ${ }^{1}$ From a practical point of view, this process is important as an alternative to the conventional combustion and due to its applications in natural-gas fueled vehicles. $^{2}$ It is also an important reaction to remove the greenhouse gas methane in automotive emissions. For these reasons, this reaction has attracted an attention already for several decades. ${ }^{1-5}$

The understanding of the kinetics of methane oxidation on $\mathrm{Pt}$ is still far from complete due to its unusual features. One of the peculiarities observed already in early studies is that under certain conditions the reaction rate measured under steady-state conditions exhibits a nearly stepwise change with varying reactant pressure. ${ }^{1}$ In particular, a two times increase of oxygen pressure can result in a five times drop in the reaction rate and then, with further increase of oxygen pressure, the reaction rate becomes independent of oxygen pressure. Another observation of the same category is that with increasing oxygen coverage the $\mathrm{CH}_{4}$ conversion exhibits a peaklike behavior, i.e., it first rapidly increases and then drops. ${ }^{3}$

Despite the peculiarities described above, the dependence of the reaction rate on reactant pressures has often been represented in the simplest power-law form,

$$
W=A P_{\mathrm{CH}_{4}}^{\alpha} P_{\mathrm{O}_{2}}^{\beta},
$$

where $A, \alpha$, and $\beta$ are constants. The corresponding data (see, e.g., Ref. 6 and/or the results collected in Table 1 of Ref. 4) indicate that the reaction is first order in $\mathrm{CH}_{4}$ and zero order in $\mathrm{O}_{2}$ at oxygen excess. For $\mathrm{CH}_{4} / \mathrm{O}_{2}$ pressure ratio above 0.3 , the reaction order in $\mathrm{CH}_{4}$ is 1 as well. The reaction order in $\mathrm{O}_{2}$ may, however, be both negative and positive.

The kinetics under consideration was also described by using the simplest Langmuir-Hinshelwood model, ${ }^{4}$ resulting in

$$
W=\frac{A K_{1} P_{\mathrm{CH}_{4}}\left(K_{2} P_{\mathrm{O}_{2}}\right)^{1 / 2}}{\left[1+K_{1} P_{\mathrm{CH}_{4}}+\left(K_{2} P_{\mathrm{O}_{2}}\right)^{1 / 2}\right]^{2}},
$$

where $A, K_{1}$, and $K_{2}$ are constants. More detailed conventional models were proposed as well. ${ }^{7}$ Although the applicability of such models is wider, they do not predict and explain the stepwise peculiarities.

One of the likely reasons of the special features of the kinetics of methane oxidation on $\mathrm{Pt}$ is surface-oxide formation. The important role of this factor in methane oxidation on Pt and Pd has long been recognized in the literature. In particular, reviewing the relevant literature (up to 1996) and comparing the catalytic activities of Pt and Pd, Burch et al. ${ }^{3}$ wrote that "the commonly accepted model" is "that platinum is more efficient in the metallic state, whereas palladium is most active in the oxidized state." Concerning the surfaceoxide formation, it is appropriate to note that methane oxidation on Pt is usually studied at relatively high temperatures (from 500 to $1100 \mathrm{~K}$ ) and the in situ infrared spectroscopy indicates that the surface is primarily covered by oxygen. ${ }^{8}$ These conditions are similar to those corresponding to a high-active reaction regime in $\mathrm{CO}$ oxidation on Pt. For the latter reaction, the influence of surface-oxide formation on the reaction kinetics has long been recognized as well (see scanning tunnel microscopy data for a single-crystal surface, ${ }^{9}$ Fourier transform infrared spectroscopy data for supported $\mathrm{Pt},{ }^{10}$ and other relevant references in Ref. 11).

Reaction kinetics including the surface-oxide formation are sometimes described by employing the mean-field Marsvan Krevelen model. ${ }^{12}$ The corresponding results are, however, similar to those predicted by the Langmuir- 
Hinshelwood model, and accordingly it cannot explain the peculiarities of methane oxidation on Pt either (for discussion of the shortcomings of the Mars-van Krevelen model, see Ref. 13). Physically, the surface oxide represents a new phase, and its formation should be described in terms of the theory of first-order phase transitions. Following this line, we recently formulated a simple lattice Monte Carlo (MC) model of surface-oxide formation and used it to simulate the kinetics of $\mathrm{CO}$ oxidation. ${ }^{11}$ In this article, employing this model, we illustrate how the surface-oxide formation may influence the kinetics of methane oxidation on Pt. In general, the experience of MC simulations of catalytic reactions accompanied by first-order phase transitions is now limited (see review, ${ }^{14}$ recent simulations, ${ }^{15}$ and references therein). For methane oxidation, such simulations are lacking.

\section{MODEL}

Simulations of methane oxidation on $\mathrm{Pt}$ with surfaceoxide formation are far from straightforward for several reasons. (i) The reaction includes many steps, and the corresponding kinetic parameters are not well established even if one ignores surface-oxide formation. (ii) The details of surface-oxide formation are not well established either [for available experiment and density functional theory (DFT) calculations, see Refs. 9, 16, and 17]. (iii) The bulk of experimental data on the kinetics of methane oxidation have been obtained on supported $\mathrm{Pt}$, and accordingly the detailed interpretation of such data is complicated by the factors specific for nanometer-sized chemistry (for a relevant general discussion, see Ref. 18). Under such circumstances, the attempts to fit in detail some of the available kinetics may easily result in misleading conclusions. For these reasons, our study is focused on the conceptual understanding of what may happen in the reaction under consideration. Specifically, we formulate a generic model, containing a minimal number of reaction steps and kinetic parameters, and focus it on the likely role of surface oxide in the reaction (a good example of this strategy is the famous Ziff-Gulari-Barshad mode ${ }^{19}$ of $\mathrm{CO}$ oxidation). Following this line, we first describe a lattice model of surface-oxide formation and then introduce the reaction steps.

According to the experiment, ${ }^{16}$ the surface oxide typically contains 2 or $3 \mathrm{ML}$ of oxygen. It becomes more stable with increasing oxygen coverage. In our simulations, we use a minimal model including two (surface and subsurface) layers of sites. Each layer is represented by a square lattice like in a cubic lattice. A site of the surface layer may be either vacant or occupied by chemisorbed oxygen, $\mathrm{O}$, or by oxide, $\mathrm{O}^{*}$. A site of the subsurface layer may be vacant or occupied by $\mathrm{O}^{*}$. To describe the phase transition, we take into account nearest-neighbor $(\mathrm{nn}) \mathrm{O}-\mathrm{O}, \mathrm{O}-\mathrm{O}^{*}$, and $\mathrm{O}^{*}-\mathrm{O}^{*}$ interactions and represent the Hamiltonian of oxygen atoms as

$$
\begin{aligned}
H= & \sum_{i}\left(E_{0} n_{i}^{(0)}+E_{1} n_{i}^{(1)}+E_{2} n_{i}^{(2)}\right)+\sum_{i, j}\left(\epsilon_{00} n_{i}^{(0)} n_{j}^{(0)}\right. \\
& +\epsilon_{01} n_{i}^{(0)} n_{j}^{(1)}+\epsilon_{02} n_{i}^{(0)} n_{j}^{(2)}+\epsilon_{11} n_{i}^{(1)} n_{j}^{(1)}+\epsilon_{12} n_{i}^{(1)} n_{j}^{(2)} \\
& \left.+\epsilon_{22} n_{i}^{(2)} n_{j}^{(2)}\right)
\end{aligned}
$$

where $\Sigma_{i}$ and $\sum_{i, j}$ are summations over sites and pairs of nn sites, $E_{0}$ and $n_{i}^{(0)}$ are the energy and site-occupation numbers for $\mathrm{O}, E_{1}, E_{2}, n_{i}^{(1)}$, and $n_{i}^{(2)}$ are the energies and occupation numbers for $\mathrm{O}^{*}$ in the surface and subsurface layers, $\epsilon_{00}, \epsilon_{01}$, and $\epsilon_{02}$ are repulsive $(>0)$ lateral and vertical $\mathrm{O}_{-}$and $\mathrm{O}_{-} \mathrm{O}^{*}$ interactions, and $\epsilon_{11}, \epsilon_{22}$ and $\epsilon_{12}$ are the attractive $(<0)$ lateral and vertical $\mathrm{O}^{*}-\mathrm{O}^{*}$ interactions.

Taking into account that one of the energies $E_{0}, E_{1}$, and $E_{2}$ can be chosen arbitrary, we use $E_{0}$ as a reference. The values of other parameters are chosen as follows: $E_{1}-E_{0}$ $=E_{2}-E_{0}=4 k_{B} T, \quad \epsilon_{00}=\epsilon_{01}=\epsilon_{02}=2 k_{B} T, \quad$ and $\epsilon_{11}=\epsilon_{12}=\epsilon_{22}$ $=-k_{B} T$. With these parameters, the adsorbed overlayer (Fig. $1)$ is in a one-phase state at $\theta<0.55 \mathrm{ML}$ and in a two-phase state at $\theta>0.55 \mathrm{ML}$, where $\theta(\theta \leqslant 2 \mathrm{ML})$ is the total oxygen coverage. (Note that the maximum possible coverage of chemisorbed oxygen is $1 \mathrm{ML}$, while it is $2 \mathrm{ML}$ for oxide oxygen.) In the former case, oxygen is in the chemisorbed state [this state is disordered at $\theta<0.33 \mathrm{ML}$ and of the $c(2$ $\times 2$ ) symmetry at $0.33<\theta<0.55 \mathrm{ML}]$ such that the $\mathrm{O}^{*}$ coverage of the surface and subsurface layers is nearly negligible. In the latter case, the $c(2 \times 2)$ chemisorbed oxygen coexists with the two-layer oxide islands.

In reality, the lateral and vertical interactions can be estimated by using the temperature-programed desorption data and are expected to be somewhat stronger than those introduced above. Our choice of the parameters is a compromise between the needs to reproduce oxygen ordering and phase separation on Pt (cf., e.g., Ref. 16) and to have computationally reasonable duration of $\mathrm{MC}$ runs.

During reaction, chemisorbed oxygen is supplied from the gas phase via irreversible (at the temperatures of interest here) dissociative adsorption on pairs of vacant nn sites,

$$
\left(\mathrm{O}_{2}\right)_{\mathrm{gas}}+2 \mathrm{Z} \rightarrow 2 \mathrm{O}_{\text {ads }}
$$

$\mathrm{O}$ is allowed to diffuse in the surface layer via jumps to vacant $\mathrm{nn}$ sites. The oxide formation and removal are represented as

$$
\mathrm{O}_{\mathrm{ads}} \rightleftharpoons \mathrm{O}_{\mathrm{ads}}^{*(1)}
$$

where $\mathrm{O}_{\mathrm{ads}}^{*(1)}$ is the oxide form of oxygen in the surface layer. $\mathrm{O}^{*}$ is assumed to diffuse in and between the layers via lateral and vertical jumps to vacant nn sites.

The reaction is considered to be limited by dissociative $\mathrm{CH}_{4}$ chemisorption occurring via the Eley-Rideal-type mechanism with breaking one of the $\mathrm{C}-\mathrm{H}$ bonds, ${ }^{21,22}$

$$
\left(\mathrm{CH}_{4}\right)_{\mathrm{gas}}+\mathrm{O}_{\mathrm{ad}} \rightarrow\left(\mathrm{CH}_{3}\right)_{\mathrm{ads}}+\mathrm{OH}_{\mathrm{ad}}
$$

or

$$
\left(\mathrm{CH}_{4}\right)_{\mathrm{gas}}+\mathrm{O}_{\mathrm{ad}}^{*} \rightarrow\left(\mathrm{CH}_{3}\right)_{\mathrm{ads}}+\mathrm{OH}_{\mathrm{ad}} .
$$

Taking into account that oxygen and $\mathrm{CH}_{3}$ are usually adsorbed on hollow and top sites, respectively, the adsorption of these species is assumed to be cooperative. Note that the use of the Eley-Rideal mechanism implies either a pure gas phase $\left(\mathrm{CH}_{4}\right)$ adsorbate $(\mathrm{O})$ reaction or one where $\mathrm{CH}_{4}$ is in a weakly bound and short lived state-it yields the same kinetics.

The other reaction steps are considered to be rapid and not treated explicitly. Practically, this means that the products 


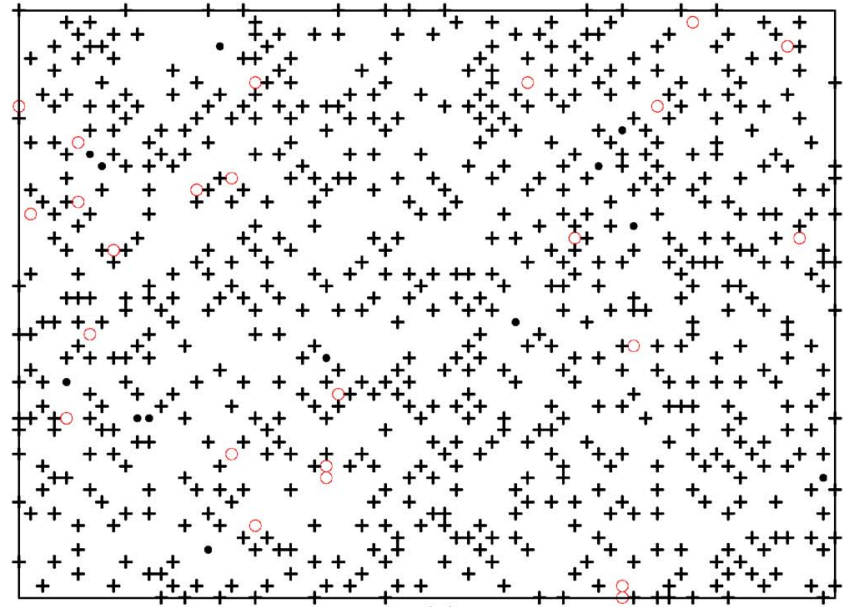

(a)

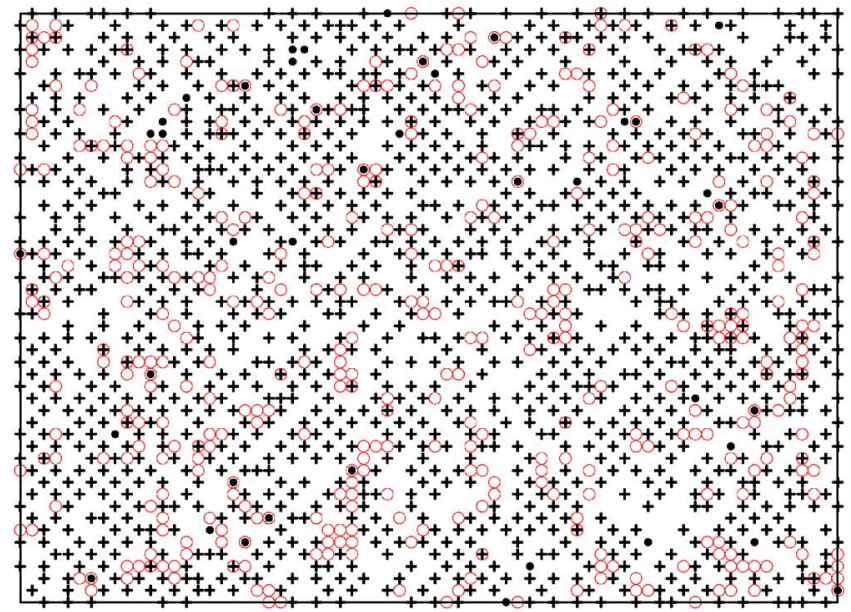

(b)

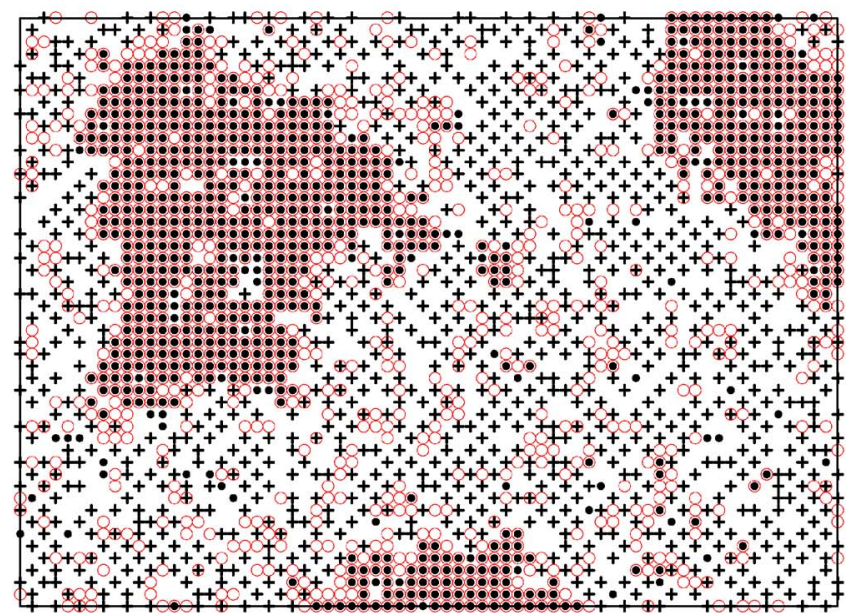

(c)

FIG. 1. (Color online) A $(50 \times 70)$ fragment of the lattice after $10^{5}$ trials of oxygen diffusion and conversion [step (5)] per site. The total oxygen coverage is (a) 0.2 , (b) 0.5 , and (c) $0.8 \mathrm{ML}$. The plus signs and filled circles show $\mathrm{O}$ and $\mathrm{O}^{*}$ in the surface layer. The open circles indicate $\mathrm{O}^{*}$ in the subsurface layer. Note that on panel (c) the surface and subsurface $\mathrm{O}^{*}$ form two-layer islands, i.e., each surface $\mathrm{O}^{*}$ as a rule contacts a subsurface $\mathrm{O}^{*}$ located below.

of reaction (6) or (7) are removed from the surface just after formation. Thus, the adsorbed overlayer contains only oxygen.
Adsorbed oxygen can desorb. The activation energy for this process is, however, high [about $55 \mathrm{kcal} / \mathrm{mol}$ (Ref. 23 and 24)], and accordingly the oxygen removal from the surface due to desorption is typically slow compared to the oxygen consumption due to reaction. In our simulations, oxygen desorption is neglected.

To describe the phase transition on the surface, we take into account the effect of lateral and vertical interactions on the rates of $\mathrm{O}$ and $\mathrm{O}^{*}$ diffusion jumps to $\mathrm{nn}$ vacant sites and the rate constants of oxide formation or removal. According to DFT calculations, ${ }^{25}$ the activation energy for oxygen diffusion on the $\mathrm{Pt}(111)$ surface is low (about $13 \mathrm{kcal} / \mathrm{mol}$; for other relevant data, see Ref. 24). This means that this process is fast. The surface-oxide formation is observed on Pt even at $425 \mathrm{~K}$. ${ }^{9}$ Thus, at temperatures typical for methane oxidation (above $500 \mathrm{~K}$ ), oxygen diffusion in surface oxide is fast as well.

For a given arrangement of $\mathrm{O}$ and $\mathrm{O}^{*}$, we represent the diffusion-jump rate constant as a product of the maximum jump rate constant $k_{\text {jump }}$ and the conventional Metropolis probability,

$$
\mathcal{P}_{M}=\left\{\begin{array}{l}
1 \text { for } \epsilon_{f} \leqslant \epsilon_{i} \\
\exp \left[-\left(\epsilon_{f}-\epsilon_{i}\right) / k_{B} T\right] \text { for } \epsilon_{f}>\epsilon_{i},
\end{array}\right.
$$

where $\epsilon_{i}$ and $\epsilon_{f}$ are the energies in the initial and final states, respectively. (If necessary, the Metropolis dynamics can be replaced by more realistic dynamics.)

In general, the maximum rate constants of $\mathrm{O}$ and $\mathrm{O}^{*}$ diffusion jumps to nn vacant sites are different. To reduce the number of kinetic parameters, we assume here that these rate constants are equal. Taking into account that $\mathrm{O}^{*}$ can perform four lateral and one vertical jumps, the maximum diffusion rate is defined as $k_{\text {dif }}=5 k_{\text {jump }}$.

For oxygen conversion (5), we use the Metropolis dynamics as well, i.e., the event rate constant is represented as a product of the maximum rate constant $k_{\mathrm{ox}}$ and the Metropolis probability.

In reality, the rates of $\mathrm{O}_{2}$ adsorption and methane dissociation [steps (4), (6), and (7)] also depend on lateral and vertical interactions. In our present $\mathrm{MC}$ simulations, this effect is neglected for simplicity, i.e., the corresponding rate constants $k_{\mathrm{ad}}, k_{r 1}$, and $k_{r 2}$ are assumed to be independent of the state of the adjacent sites (this approximation is relaxed in Sec. V). Taking into account that on Pt the oxide is less active than the chemisorbed oxygen in methane oxidation, we use $k_{r 1}>k_{r 2}$.

\section{ALGORITHM OF SIMULATIONS}

MC simulations are now widely used to analyze the kinetics of catalytic heterogeneous reactions. ${ }^{14,20}$ In such simulations, the dimensionless probabilities of elementary events are usually obtained by normalizing the corresponding rate constants to the properly defined maximum rate constant $k_{\max }$. In our simulations, we use $k_{\max }=k_{\mathrm{ad}} P_{\mathrm{O}_{2}}+k_{r 1} P_{\mathrm{CH}_{4}}$ $+2\left(k_{\mathrm{dif}}+k_{\mathrm{ox}}\right)$ (the factor 2 takes into account that diffusion occurs in two layers). To characterize the relative rate of the catalytic cycle, we introduce the probability $p_{\text {cyc }}=\left(k_{\mathrm{ad}} P_{\mathrm{O}_{2}}\right.$ 
$\left.+k_{r 1} P_{\mathrm{CH}_{4}}\right) / k_{\max }$. To characterize the relative rate of $\mathrm{CH}_{4}$ reaction inside the catalytic cycle, we employ the parameter $p=k_{r 1} P_{\mathrm{CH}_{4}} /\left(k_{\mathrm{ad}} P_{\mathrm{O}_{2}}+k_{r 1} P_{\mathrm{CH}_{4}}\right)$.

The simulations are performed on a two-layer $L \times L$ lattice with periodic boundary conditions. The MC runs consist of sequential trials to realize elementary events. Specifically, a trial of the catalytic cycle or diffusion/oxidation is performed as described in items 1 and 2 provided, respectively, that $\rho<p_{\text {cyc }}$ and $\rho>p_{\text {cyc }}$, where $\rho(0<\rho \leqslant 1)$ is a random number.

(1) For a catalytic cycle, a site in the surface layer is chosen at random and a trial of $\mathrm{CH}_{4}$ reaction or $\mathrm{O}_{2}$ adsorption is performed, respectively, if $\rho<p$ or $\rho>p$, where $\rho$ is an another random number. The $\mathrm{CH}_{4}$-reaction trial is executed with unit probability if the site is occupied by $\mathrm{O}$, with probability $k_{r 2} / k_{r 1}$ if the site is occupied by $\mathrm{O}^{*}$, and terminated if the site is vacant. If the reaction trial is successful, $\mathrm{O}$ or $\mathrm{O}^{*}$ is removed from the surface and simultaneously three other $\mathrm{O}$ and/or $\mathrm{O}^{*}$, randomly chosen in the surface layer, are removed as well in order to fulfill the balance equation (1) [this procedure mimics rapid reaction steps occurring after step (6) or (7), and it does not discriminate between $\mathrm{O}$ and $\left.\mathrm{O}^{*}\right]$. The $\mathrm{O}_{2}$-adsorption trial is executed if the site is vacant and terminated if the site is occupied. In the $\mathrm{O}_{2}$-adsorption case, one of the nn sites in the surface layer is selected at random, and the trial is considered to be successful if the latter site is vacant.

(2) For diffusion, oxidation $\left(\mathrm{O} \rightarrow \mathrm{O}^{*}\right)$ or reduction $\left(\mathrm{O}^{*}\right.$ $\rightarrow \mathrm{O}$ ), a site in one of the layers is chosen at random. If the site is vacant, the trial ends. If the site is occupied by $\mathrm{O}$, the trial of diffusion or oxide formation is performed with probabilities $k_{\mathrm{dif}} \mathcal{P}_{M} /\left(k_{\mathrm{dif}}+k_{\mathrm{ox}}\right)$ and $k_{\mathrm{ox}} \mathcal{P}_{M} /\left(k_{\mathrm{dif}}+k_{\mathrm{ox}}\right)$ (for diffusion, one of the five nn sites is chosen at random, and the trial is executed if the latter site is vacant and belongs to the surface layer). If the site is occupied by $\mathrm{O}^{*}$ and belongs to the surface layer, the trial of diffusion or oxide removal is performed with probabilities $k_{\mathrm{dif}} \mathcal{P}_{M} /\left(k_{\mathrm{dif}}+k_{\mathrm{ox}}\right)$ and $k_{\mathrm{ox}} \mathcal{P}_{M} /\left(k_{\mathrm{dif}}+k_{\mathrm{ox}}\right)$ (for diffusion, one of the five nn sites is chosen at random, and the trial is executed if the latter site is vacant). If the site is occupied by $\mathrm{O}^{*}$ and belongs to the subsurface layer, the trial of diffusion is performed with probability $k_{\text {dif }} \mathcal{P}_{M} /\left(k_{\text {dif }}+k_{\text {ox }}\right)$ (for this trial, one of the five nn sites is chosen at random, and the trial is executed if the latter site is vacant).

To measure time, we use the so-called MC steps (MCS). To relate MC time to the rate of the catalytic cycle, one MCS is identified with $L^{2} / p_{\text {cyc }} \mathrm{MC}$ trials. In this case, the MC and real times are interconnected as $t_{\mathrm{MC}}=k_{\mathrm{cyc}} t$.

\section{RESULTS OF SIMULATIONS}

The simulations were performed on a lattice with $L$ $=200$. The rate constants $k_{\mathrm{ox}}$ and $k_{\text {jump }}$ were set equal (in reality, $k_{\text {jump }}$ may be larger than $k_{\mathrm{ox}}$, but it does not change the conclusions). Step (6) was considered to be 20 times faster than step (7) (i.e., $\left.k_{r 2} / k_{r 1}=1 / 20\right)$. For the catalytic
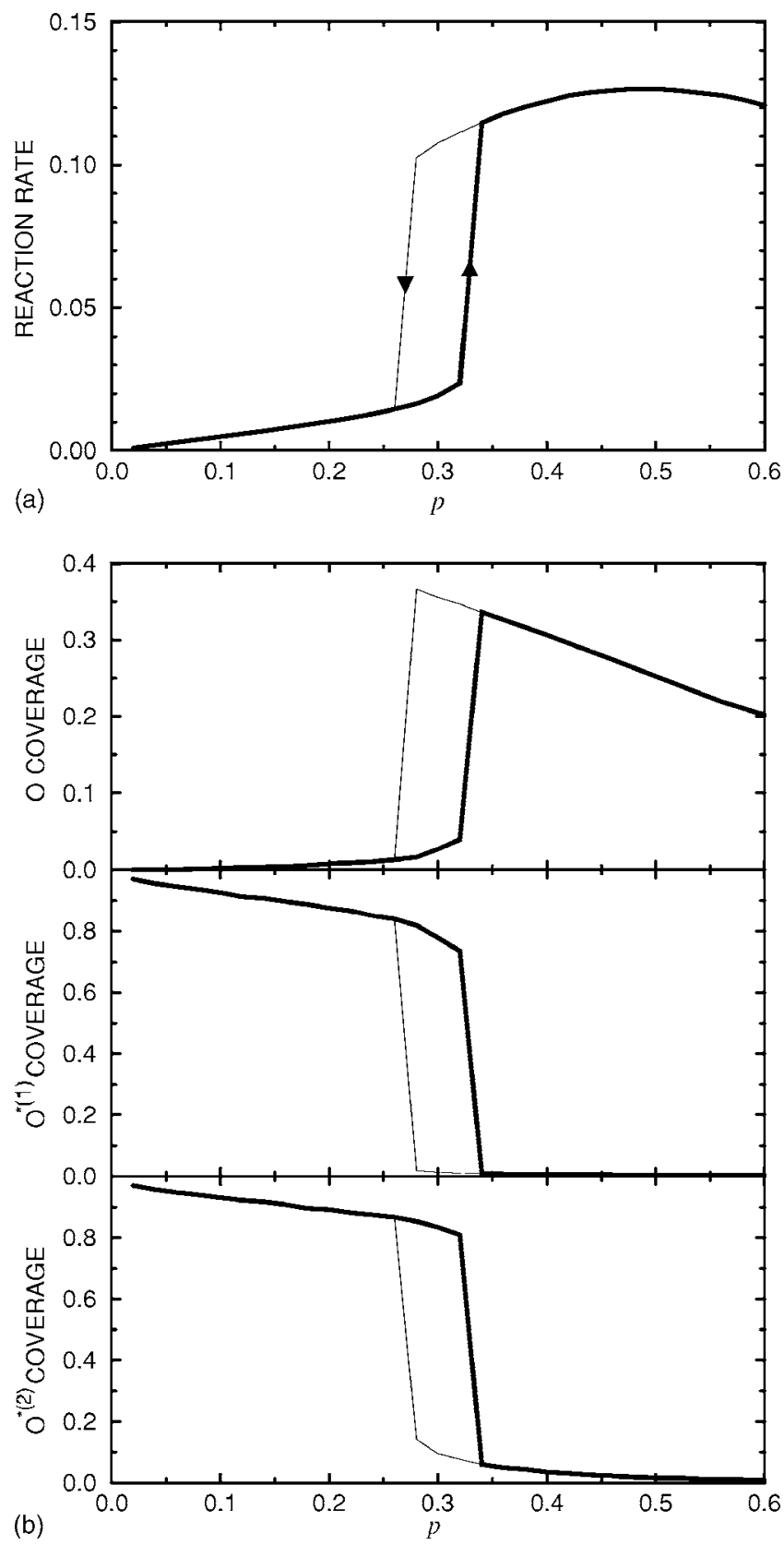

FIG. 2. (a) Reaction rate (per MCS) and (b) $\mathrm{O}, \mathrm{O}^{*(1)}$, and $\mathrm{O}^{*(2)}$ coverages as a function of $p$.

cycle, we used $p_{\text {cyc }}=1 / 200$ (this means that the catalytic cycle was two orders of magnitude slower than $\mathrm{O}$ or $\mathrm{O}^{*}$ diffusion). $p$ was employed as a governing parameter. During MC runs, $p$ was increased from 0.02 up to 0.6 and then decreased down to 0.02 step by step by 0.02 . The time interval between the steps was chosen to be $200 \mathrm{MCS}$. The data points were obtained during the last 100 MCS of each step.

The key results of our simulations are shown in Fig. 2. At low values of $p$, oxygen is in excess, and the surface is seen to be almost completely covered by oxide. In this case, the reaction occurs primarily on oxide, and accordingly the reaction rate is given by

$$
W \simeq k_{r 2} P_{\mathrm{CH}_{4}} .
$$

Thus, the reaction is first order in $\mathrm{CH}_{4}$ and zero order in $\mathrm{O}_{2}$. With increasing $p$, this regime is maintained up to $p=0.32$. 
At this point, there is a stepwise transition from the state where the surface covered by oxide to the state where the surface is covered by chemisorbed oxygen. This transition results in a stepwise increase of the reaction rate because chemisorbed oxygen is more active in the reaction. With further increase of $p$, the oxygen coverage decreases but this decrease is compensated by increase of the $\mathrm{CH}_{4}$ impingement rate, and accordingly the reaction rate is nearly constant.

With decreasing $p$ back from 0.6 to 0.02 , the reaction kinetics is basically the same. The only difference is that the stepwise transition from the high-reactive state to the lowreactive state takes place at $p=0.28$. Thus, as one could expect, the model predicts hysteresis. The hysteresis loop is, however, relatively narrow. With increasing/decreasing diffusion rate, the hysteresis loop becomes narrower/wider (not shown). In experiments, a broad hysteresis was observed in the temperature dependence of the reaction rate on $\mathrm{Pt}$ (Ref. 26) and $\mathrm{Pd},{ }^{27}$ and it was attributed to the oxide formation. ${ }^{27}$ Our model can be used to describe the latter hysteresis. Practically, it is, however, rather difficult because with variation of temperature the rate constants of elementary reaction steps change in a very broad range, and accordingly there is no guarantee that the MC simulations are feasible.

Scrutinizing Fig. 2(b), one can note that during the stepwise transitions the coverage of the surface by chemisorbed oxygen is about 0.35 . This value is lower than that $(\simeq 0.55)$ corresponding to the phase transition from the $c(2 \times 2)$ state to the $c(2 \times 2)+$ oxide state. This means that although the stepwise transitions shown in Fig. 2 are directly related to the chemisorbed $\rightleftharpoons$ oxide phase transition, the corresponding critical value of $\mathrm{O}$ coverage $(\simeq 0.35)$ is determined by the interplay of reaction and phase transition. In additional simulations (not shown), we have proved that with appreciable increase of the diffusion rate the latter value gradually increases towards 0.55 .

Finally, it is appropriate to discuss in more detail the high-reactive state. In this state, the surface is covered primarily by chemisorbed oxygen and as already noted the oxygen coverage $\theta_{\mathrm{O}}$ is relatively small (below $\simeq 0.35$ ) and accordingly oxygen is disordered. Under such circumstances, the balance of the reaction and adsorption rates is described as

$$
k_{r 1} P_{\mathrm{CH}_{4}} \theta_{\mathrm{O}} \simeq 2 k_{\mathrm{ad}} P_{\mathrm{O}_{2}}\left(1-\theta_{\mathrm{O}}\right)^{2},
$$

where 2 is the coefficient related to the reaction stoichiometry [Eq. (1)]. Taking into account that $\theta_{\mathrm{O}}$ is relatively small and replacing $\left(1-\theta_{\mathrm{O}}\right)^{2}$ by $\left(1-2 \theta_{\mathrm{O}}\right)$ in the right-hand part of Eq. (2), we get $\theta_{\mathrm{O}} \simeq 2 k_{\mathrm{ad}} P_{\mathrm{O}_{2}} /\left(k_{r 1} P_{\mathrm{CH}_{4}}+4 k_{\mathrm{ad}} P_{\mathrm{O}_{2}}\right)$, and accordingly,

$$
W \simeq \frac{2 k_{r 1} k_{\mathrm{ad}} P_{\mathrm{CH}_{4}} P_{\mathrm{O}_{2}}}{k_{r 1} P_{\mathrm{CH}_{4}}+4 k_{\mathrm{ad}} P_{\mathrm{O}_{2}}} .
$$

The latter expression indicates that the reaction orders in $\mathrm{CH}_{4}$ and $\mathrm{O}_{2}$ are positive. The values of the reaction orders are, however, poorly defined.

In a more general context, the reaction rate constants in Eq. (10) are expected to strongly depend on coverage due to lateral interactions. Using the simplest mean-field expressions, $k_{r 1} \propto \exp \left(-\alpha \theta_{\mathrm{O}}\right)$ and $k_{\mathrm{ad}} \propto \exp \left(-\beta \theta_{\mathrm{O}}\right)$, one can easily obtain

$$
W \propto P_{\mathrm{CH}_{4}}^{\alpha /(\alpha+\beta)} P_{\mathrm{O}_{2}}^{\beta /(\alpha+\beta)} .
$$

\section{CONCLUSION}

We have presented a generic model of methane oxidation on $\mathrm{Pt}$ with the emphasis on the role of surface-oxide formation. The latter process has been treated in terms of the theory of first-order phase transitions. The corresponding MC simulations indicate that the surface-oxide formation may result in stepwise features in the reaction kinetics. Specifically, with increasing $\mathrm{CH}_{4}$ pressure and/or decreasing $\mathrm{O}_{2}$ pressure, the model predicts a sharp transition from the lowreactive state with the surface completely covered by oxide to the high-reactive state with the surface covered by chemisorbed oxygen. In the former case, the reaction is first order in $\mathrm{CH}_{4}$ and zero order in $\mathrm{O}_{2}$. In the latter case, both reaction orders are positive.

Our model qualitatively explains the peculiarities of the kinetics of methane oxidation on Pt. For example, already early experiments ${ }^{1}$ indicated that with increasing oxygen pressure the reaction rate may suddenly drop and then to be independent of oxygen pressure. According to our model, such observations can be related to a transition from the state with the surface covered by chemisorbed oxygen to the state with the surface covered by oxide. Our model also explains why the reaction orders measured under slightly different conditions are often quite different.

\section{ACKNOWLEDGMENTS}

This work has been performed within the Competence Centre for Catalysis, which is hosted by Chalmers University of Technology and financially supported by the Swedish Energy Agency and the member companies: AB Volvo, Volvo Car Corporation, Scania CV AB, GM Powertrain Sweden AB, Haldor Topsoe A/S, and the Swedish Space Agency. The authors thank M. Skoglundh for useful discussions.

${ }^{1}$ J. G. Firth and H. B. Holland, Trans. Faraday Soc. 65, 1121 (1969).

${ }^{2}$ P. Gelin, L. Urfels, M. Primet, and E. Tena, Catal. Today 83, 45 (2003).

${ }^{3}$ R. Burch, P. K. Loader, and F. J. Urbano, Catal. Today 27, 243 (1996).

${ }^{4}$ L. Ma, D. L. Trimm, and C. Jiang, Appl. Catal., A 138, 275 (1996).

${ }^{5}$ P.-A. Carlsson, E. Fridell, and M. Skoglundth, Catal. Lett. 115, 1 (2007).

${ }^{6}$ Y.-F. Y. Yao, Ind. Eng. Chem. Prod. Res. Dev. 19, 293 (1980); D. L. Trimm and C.-W. Lam, Chem. Eng. Sci. 35, 1405 (1980); M. Niwa, K. Awano, and Y. Murakami, Appl. Catal. 7, 317 (1983); K. Otto, Langmuir 5, 1364 (1989).

${ }^{7}$ P. Aghalayam, Y. K. Park, N. Fernandes, V. Papavassiliou, A. B. Mhadeshwar, and D. G. Vlachos, J. Catal. 213, 23 (2003).

${ }^{8}$ A. Bourane, C. Cao, and K. L. Hohn, Appl. Catal., A 302, 224 (2006).

${ }^{9}$ B. L. M. Hendriksen and J. W. M. Frenken, Phys. Rev. Lett. 89, 046101 (2002).

${ }^{10}$ P.-A. Carlsson, V. P. Zhdanov, and M. Skoglundh, Phys. Chem. Chem. Phys. 8, 2703 (2006).

${ }^{11}$ V. P. Zhdanov, J. Chem. Phys. 126, 074706 (2007).

${ }^{12}$ P. Mars and D. W. van Krevelen, Chem. Eng. Sci. 3, 41 (1954).

${ }_{14}^{13}$ M. A. Vannice, Catal. Today 123, 18 (2007).

${ }^{14}$ V. P. Zhdanov, Surf. Sci. Rep. 45, 231 (2002).

${ }^{15}$ V. P. Zhdanov and T. Matsushima, Phys. Rev. Lett. 98, 036101 (2007).

${ }^{16}$ J. F. Weaver, J.-J. Chen, and A. L. Gerrard, Surf. Sci. 592, 83 (2005). 
${ }^{17}$ T. M. Pedersen, W. X. Li, and B. Hammer, Phys. Chem. Chem. Phys. 8, 1566 (2006).

${ }^{18}$ V. P. Zhdanov and B. Kasemo, Surf. Sci. Rep. 39, 25 (2000).

${ }^{19}$ R. M. Ziff, E. Gulari, and Y. Barshad, Phys. Rev. Lett. 56, 2553 (1986).

${ }^{20}$ H. C. Kang and W. H. Weinberg, Chem. Rev. (Washington, D.C.) 95, 667 (1995); D. J. Liu and J. W. Evans, Multiscale Model. Simul. 4, 424 (2005); A. Chatterjee and D. G. Vlachos, J. Comput.-Aided Mater. Des. 14, 253 (2007).
${ }^{21}$ R. Burch and T. C. Watling, J. Catal. 169, 45 (1997).

${ }^{22}$ V. P. Zhdanov and B. Kasemo, J. Catal. 195, 46 (2000).

${ }^{23}$ D. H. Parker, M. E. Bartram, and B. E. Koel, Surf. Sci. 217, 489 (1989).

${ }^{24}$ V. P. Zhdanov and B. Kasemo, Surf. Sci. 415, 403 (1998).

${ }^{25}$ A. Bogicevic, J. Strömquist, and B. I. Lundqvist, Phys. Rev. B 57, R4289 (1998).

${ }^{26}$ D. L. Trimm and C.-W. Lam, Chem. Eng. Sci. 35, 1405 (1980).

${ }^{27}$ P. Salomonsson, S. Johansson, and B. Kasemo, Catal. Lett. 33, 1 (1995). 